\title{
Sphingosine-1-phosphate protects against bisphosphonate-induced HUVEC cell death via regulation of $\mathrm{c}-\mathrm{Jun}-\mathrm{N}$-terminal kinase signaling
}

\author{
YOU-JIN LEE ${ }^{1}$, JAE-KYO JEONG ${ }^{1}$, JU-HEE LEE ${ }^{1}$, YANG-GYU PARK ${ }^{1}$, JI-HONG MOON ${ }^{1}$, \\ JAE-WON SEOL ${ }^{1}$, CHRISTOPHER J. JACKSON ${ }^{2}$ and SANG-YOUEL PARK ${ }^{1}$ \\ ${ }^{1}$ Biosafety Research Institute, College of Veterinary Medicine, Chonbuk National University, Jeonju, \\ Jeonbuk 561-756, Republic of Korea; ${ }^{2}$ Institute of Bone and Joint Research, University of Sydney, \\ Kolling Institute, Royal North Shore Hospital, St. Leonards, NSW 2065, Australia
}

Received November 9, 2012; Accepted December 27, 2012

DOI: $10.3892 /$ ijmm.2013.1266

\begin{abstract}
Bisphosphonates (BPs) remain the most widely used and effective antiresorptive agents in the treatment of postmenopausal osteoporosis. In particular, nitrogen-containing BPs (N-BPs) are more potent at inhibiting bone resorption in vivo than simple BPs, but they are associated with a number of side-effects including increased endothelial cell apoptosis in patients with multiple myeloma. Sphingosine-1-phosphate (S1P), a sphingolipid metabolite, plays important roles in the regulation of cell growth, differentiation and programmed cell death as a multifunctional bioactive lipid mediator. The aim of this study was to elucidate the protective effect and the possible mechanism of S1P against BP-induced cell damage using human umbilical vein endothelial cells (HUVECs). HUVECs were treated with S1P for $1 \mathrm{~h}$ and then with BP including alendronate, zoledronate and risedronate. S1P protects HUVECs against BP-induced cell death and the protective effect was increased by S1P in a dose-dependent manner. S1P blocked BP-induced caspase- 3 activation, nuclear factor- $\kappa \mathrm{B}$ activation, c-Jun-N-terminal kinase (JNK) phosphorylation and DNA fragmentation. The blocking of JNK phosphorylation inhibited BP-induced caspase activation and HUVEC cell death. The present study demonstrates that S1P inhibits BP-induced endothelial cell death via regulation of JNK phosphorylation, and also suggests that S1P has the potential to be a therapeutic drug in various vascular diseases induced by BP.
\end{abstract}

\section{Introduction}

Bisphosphonates (BPs) remain the most widely used and effective antiresorptive agents for the treatment of diseases in which

Correspondence to: Professor Sang-Youel Park, Biosafety Research Institute, College of Veterinary Medicine, Chonbuk National University, Dukjin Dong 664-14, Jeonju, Jeonbuk 561-756, Republic of Korea E-mail: sypark@chonbuk.ac.kr

Key words: sphingosine-1-phosphate, endothelial cell, bisphosphonate, c-Jun-N-terminal kinase there is an increase in the number or activity of osteoclasts, including tumor-associated osteolysis and hypercalcemia (1). BPs are a class of pharmacological compounds that are used in the treatment of postmenopausal osteoporosis; however, they are associated with a number of side-effects such as osteonecrosis of the jaw. Following BP administration, there was an increase in circulating endothelial cell apoptosis in multiple myeloma patients and osteonecrosis subjects (2).

Nitrogen-containing bisphosphonate (N-BP) has been reported to induce antiproliferative and apoptotic effects in human pancreatic cancer cells in vitro (3) and to exert antiangiogenic effects in vivo and in vitro (4). Alendronate (Aln) acts on bone marrow stromal cells to stimulate osteogenic differentiation and to inhibit adipogenic differentiation in a dose-dependent manner; these effects are mediated by activating extracellular signal-regulated kinase (ERK) and c-Jun-N-terminal kinase (JNK) (5). In addition, Aln inhibits the cell survival pathway stimulated by the phosphoinositide 3-kinase (PI3K)/Akt/nuclear factor- $\mathrm{\kappa B}(\mathrm{NF}-\mathrm{\kappa B})$ pathway, thus causing apoptosis of osteosarcoma cells (6). Zoledronate (Zol) inhibits human umbilical vein endothelial cell (HUVEC) adhesion, survival, migration and actin stress fiber formation by interfering with protein prenylation and it affects ERK1/2, JNK, Rock, FAK and PKB as kinases in a prenylation-dependent manner (7); it also downregulates vascular endothelial growth factor, endothelial nitric oxide synthase, Akt, and matrix metalloproteinase (MMP)-9 activities in ischemic tissues (8).

Sphingolipid metabolites play key roles in the regulation of numerous cellular processes important for health and disease. One of the most significant of these metabolites is sphingosine-1-phosphate (S1P). This signaling molecule regulates cell growth and suppresses apoptosis (9), suggesting that it may play a role in cancer. The biological effects of S1P are mediated by activation of G protein-coupled S1P receptors, leading to the activation of downstream effectors such as p44/42, MAPK and Akt (10). S1P has been shown to regulate the migratory behavior of osteoclast precursors in bone tissues (11), and it regulates a wide range of cellular activities involved in angiogenesis, wound healing, apoptosis, and atherosclerosis in vascular endothelial cells (12). Thus, 
S1P induces cell migration, expression of several cell adhesion molecules, DNA synthesis and cell survival.

We previously showed that $\mathrm{BP}$ was able to induce cell death in normal endothelial cells and activated protein C (APC) was able to inhibit BP-mediated endothelial cell damage via inhibition of NF- $\mathrm{KB}$ and enhancement of MMP-2 activity (13). In the present study, we investigated the protective effect of S1P and the possible mechanism against BP-induced endothelial cell damage in HUVECs.

\section{Materials and methods}

Cell culture. The HUVECs were purchased from Lonza (Walkersville, MD, USA). Cells were grown in Biorich containing $20 \%$ fetal bovine serum (FBS) with $50 \mu \mathrm{g} / \mathrm{ml}$ endothelial cell growth supplement (BD Biosciences) and $50 \mu \mathrm{g} / \mathrm{ml}$ heparin (Sigma). Cells were cultured at $37^{\circ} \mathrm{C}$ in a humidified atmosphere of $5 \% \mathrm{CO}_{2}$ and $95 \%$ air. HUVECs were used at passages 3-8.

Lactate dehydrogenase (LDH) assay. Cytotoxicity was assessed by the LDH assay in the supernatant medium using an LDH Cytotoxicity Detection kit (Takara Bio, Inc., Tokyo, Japan) according to the manufacturer's protocol. The LDH activity was determined by measuring the absorbance at $490 \mathrm{~nm}$ using a microplate reader (Spectra Max M2; Molecular Devices, USA).

Cell viability assay. Cell survival was counted by Guava easyCyte HT (Millipore). Cells were stained by Annexin V in the detached cells using an Annexin V assay kit (Santa Cruz Biotechnology, Inc.,) according to the manufacturer's protocol. Annexin $\mathrm{V}$ measurement was determined by measuring the fluorescence at excitation $488 \mathrm{~nm}$ and emission 525/30 using a Guava easyCyte HT (Millipore).

Protein extraction and western blot analyses. HUVECs were washed two times with PBS and lysed in a cell lysis buffer (25 mM HEPES; pH 7.4, $100 \mathrm{mM} \mathrm{NaCl}, 1 \mathrm{mM}$ EDTA, $5 \mathrm{mM}$ $\mathrm{MgCl}_{2}, 0.1 \mathrm{mM}$ DTT and protease inhibitor mixture). Proteins were electrophoretically resolved on a $10-15 \%$ sodium dodecyl sulfate (SDS) gel, and immunoblotting was performed in a routine manner. Equal amounts of lysate protein were resolved on a $10-15 \%$ SDS-polyacrylamide gel and electrophoretically transferred to a nitrocellulose membrane. Immunoreactivity was detected through sequential incubation with horseradish peroxidase-conjugated secondary antibodies and ECL reagents. The antibodies used for immunoblotting were caspase-3, phospho-JNK (Cell Signaling Technology), Rap-1A (Santa Cruz Biotechnology, Inc.,) and $\beta$-actin (Sigma). Images were examined using an Fusion-FX7 imaging system (Vilber Lourmat).

Immunofluorescent staining. Cultured HUVECs on 8-chamber culture slides were fixed with cold acetone and blocked by 5\% FBS in TBST and incubated with mouse p-JNK antibody (Cell Signaling Technology) and rabbit active caspase-3 antibody (R\&D Systems) overnight at $4^{\circ} \mathrm{C}$. After washing with TBST, cells were incubated with goat anti-mouse IgG conjugated with Alexa Fluor ${ }^{\circledR} 488$ (green) and goat anti-rabbit IgG conjugated with Alexa Fluor ${ }^{\circledR} 546$ (red). Cells were washed with TBST, mounted with fluorescence mounting medium (Dako) and observed under a fluorescence microscope (Nikon Eclipse 80i; Nikon Corporation). Images were acquired and processed using a Nikon digital camera and software (Diagnostic Instruments, Australia) and ImageJ.

Statistical analyses. Data are expressed as the means \pm standard deviation (SD), and were compared using the Student's t-test and the ANOVA Duncan test with the SAS statistical package (SAS Institute, Cary, NC, USA). The results were considered to indicate statistically significant differences at $\mathrm{P}<0.05$ or $\mathrm{P}<0.01$, and $\mathrm{P}<0.01$ or $\mathrm{P}<0.005$.

\section{Results}

$S 1 P$ protects BP-induced endothelial cell death. It is well known that BP induces endothelial cell death (13). Since S1P has been described to induce cell survival, in the present study we examined whether S1P could protect BP-induced endothelial cell death. HUVECs were exposed to Aln dosedependently for $24 \mathrm{~h}$ prior to assessment of cell viability. Aln induces a decrease in cell viability up to $50 \%$ (Fig. 1A and B) and an increase in cytotoxicity (Fig. 1C). When HUVECs were pretreated with S1P for $1 \mathrm{~h}$ and then exposed to Aln $(50 \mu \mathrm{M})$, S1P significantly prevented Aln-induced endothelial cell death in a dose-dependent manner (Fig. 1D and E). Examination of cell morphology also supported the protective effect of the S1P and Aln cotreatment in HUVECs (Fig. 1D). HUVECs were pretreated with or without $0.5 \mu \mathrm{M} \mathrm{S1P}$ and then exposed to Aln $(50 \mu \mathrm{M}), \mathrm{Zol}(100 \mu \mathrm{M})$ or risedronate (Ris) $(100 \mu \mathrm{M})$ for $24 \mathrm{~h}$. Zol and Ris treatments induced cell death as well as Aln and were significantly reversed by treatment with S1P (Fig. 2A and B). To determine the ability of S1P to inhibit BP-induced apoptosis, TUNEL assays were performed following exposure of BP in the presence or absence of S1P (Fig. 2C). A high amount of apoptotic cells was observed in BP-treated cells but S1P pretreatment inhibited cell damage by reducing the amount of apoptotic cells. Overall, we found that the order of potency of N-BPs for inducing apoptosis of HUVECs and S1P could inhibit BP-mediated endothelial cell damage.

Inhibitory effect of SIP on BP-induced cleaved caspase-3 and $N F-\kappa B$ activation in endothelial cells. Since N-BPs induced accumulation of a toxic by preventing protein prenylation, unprenylated protein increase for cell has been confirmed using Rap-1A, a geranylgeranylation marker that binds to the nongeranylgeranylated form. To evaluate whether BP accumulates cytotoxicity by protein unprenylation, HUVECs were treated with Aln in a dose-dependent manner for $24 \mathrm{~h}$ following treatment with or without $0.5 \mu \mathrm{M} \mathrm{S1P}$ and then an experiment to measure Rap-1A was performed. We found that $25 \mu \mathrm{M}$ of Aln inhibited geranylgeranylation slightly and $50 \mu \mathrm{M}$ of Aln did so strongly, whereas cotreatment with S1P and Aln reduced the unprenylated form of Rap-1A protein compared to Aln treatment alone (Fig. 3A and B).

Caspases play a key role in the execution phase of apoptosis. Caspase-3 subsequently cleaved cellular substrates, thereby precipitating the marked morphological changes of apoptosis. Herein, we assessed whether S1P has inhibitory effects on 


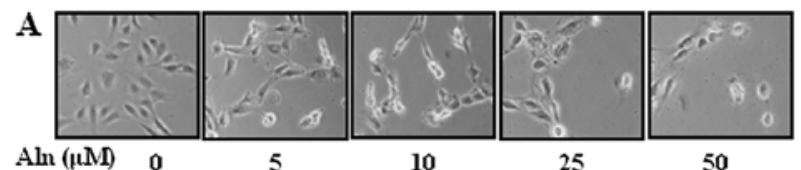

B

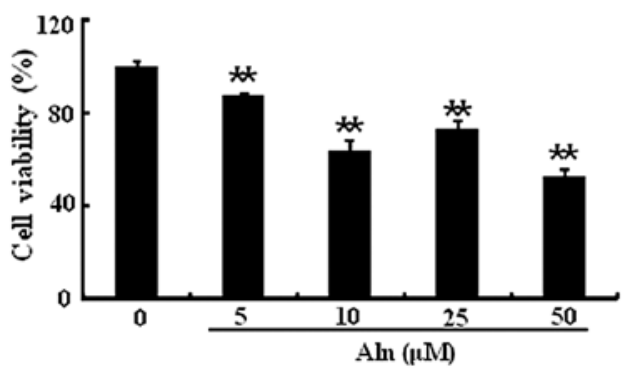

C

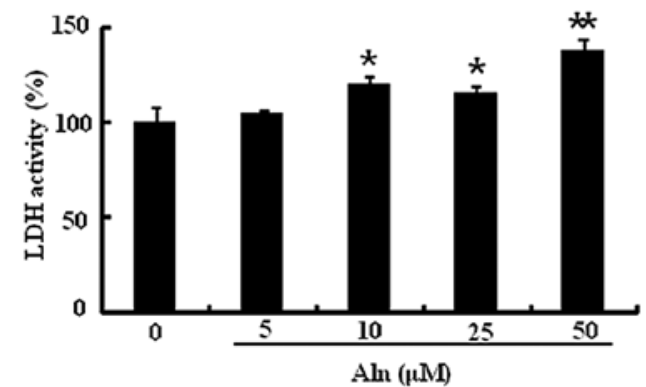

D
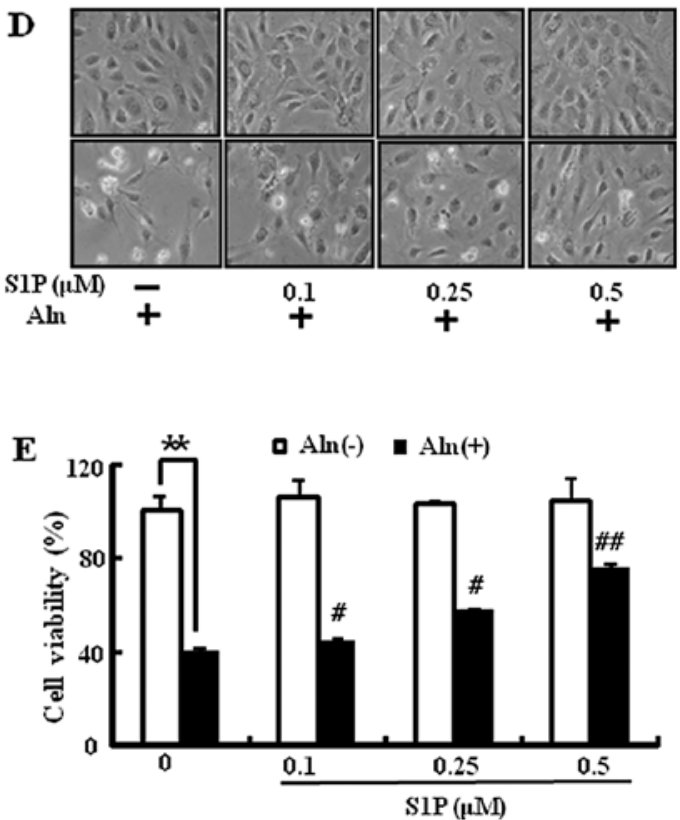

Figure 1. Administration of S1P prevented HUVECs from Aln-induced cell death. HUVECs were treated with Aln for $24 \mathrm{~h}$ in a dose-dependent manner. (A) Cell morphology was photographed with a light microscope (x200). (B) Cell viability was measured after Annexin V assay by flow cytometry. (C) Cells were exposed to Aln, and release of LDH into the cell culture supernatant from damaged cells was measured. (D) HUVECs were pretreated with S1P for $1 \mathrm{~h}$ in a dose-dependent manner and exposed to $500 \mu \mathrm{M}$ Aln for $24 \mathrm{~h}$. Cell morphology was photographed with a light microscope (x200). (E) Cell viability was measured by Annexin $\mathrm{V}$ assay. The experiments were repeatedly performed to confirm the results. The data were analyzed using analysis of variance (ANOVA) and Duncan multiple range. Bar graph indicates the mean $\pm \mathrm{SD}(\mathrm{n}=3) .{ }^{*} \mathrm{P}<0.05,{ }^{* *} \mathrm{P}<0.01$, significant differences between control and each treatment group. ${ }^{\#} \mathrm{P}<0.01,{ }^{\# \#} \mathrm{P}<0.005$, significant differences between $500 \mu \mathrm{M}$ Aln and cotreatment with S1P group. S1P, sphingosine-1-phosphate; Aln, alendronate.

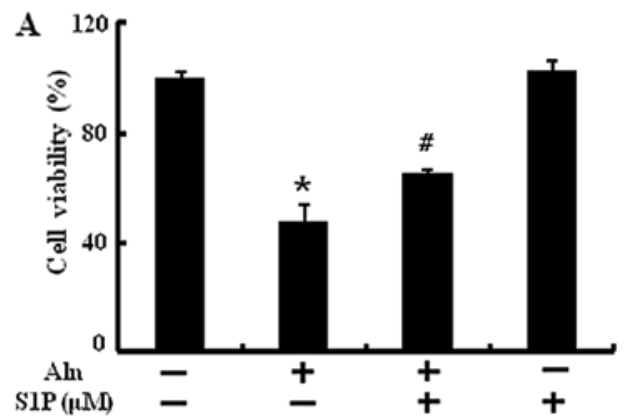

C

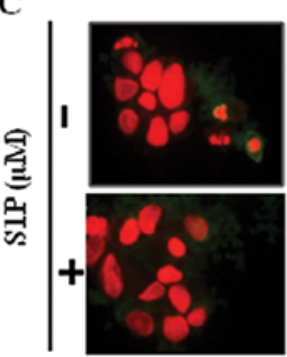

Con
B

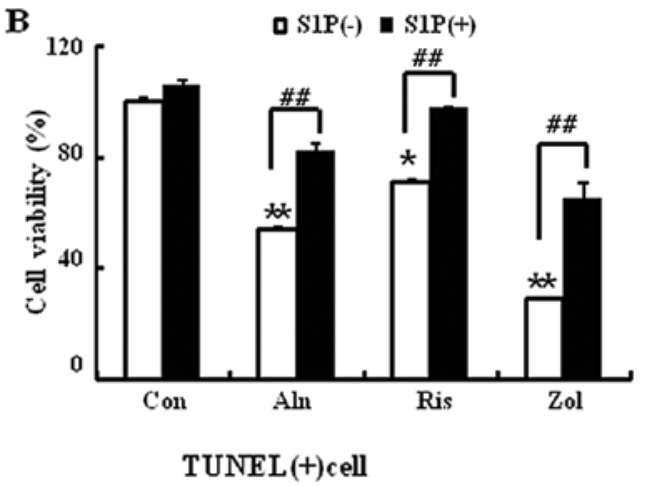

$(+)$ cell

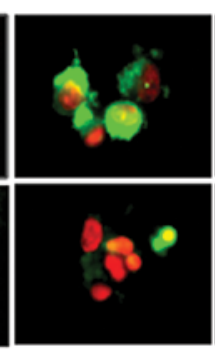

Aln

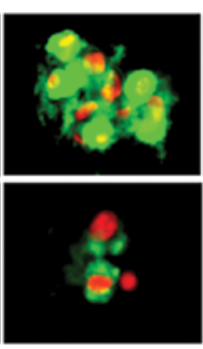

Ris

Figure 2. S1P protects against BP-induced endothelial cell death. HUVECs were pretreated with $0.5 \mu \mathrm{M}$ of S1P $(1 \mathrm{~h})$ and then exposed to $500 \mu \mathrm{M}$ Aln for $24 \mathrm{~h}$. (A) Cell viability was measured after Annexin V assay by flow cytometry. (B) Cells were pretreated with $0.5 \mu \mathrm{M}$ of S1P (1 h) and then exposed to Aln $(500 \mu \mathrm{M})$, Zol $(100 \mu \mathrm{M})$ or Ris $(100 \mu \mathrm{M})$ and cell viability was measured. (C) Representative immunofluorescence images of TUNEL-positive (green) HUVECs at $12 \mathrm{~h}$ after exposure to $500 \mu \mathrm{M}$ of Aln in the absence and presence of S1P. The cells were counterstained with propidium iodide (red) to show all cell nuclei. Magnification, $x 400$. Bar graph indicates the mean $\pm \mathrm{SEM}(\mathrm{n}=3)$. ${ }^{*} \mathrm{P}<0.05,{ }^{* *} \mathrm{P}<0.01$, significant differences between control and each treatment group. ${ }^{\#} \mathrm{P}<0.01,{ }^{\# \#} \mathrm{P}<0.005$, significant differences between $500 \mu \mathrm{M}$ Aln and cotreatment with S1P group. BP, bisphosphonate; S1P, sphingosine-1-phosphate; Aln, alendronate; Ris, risedronate; Zol, zoledronate. 

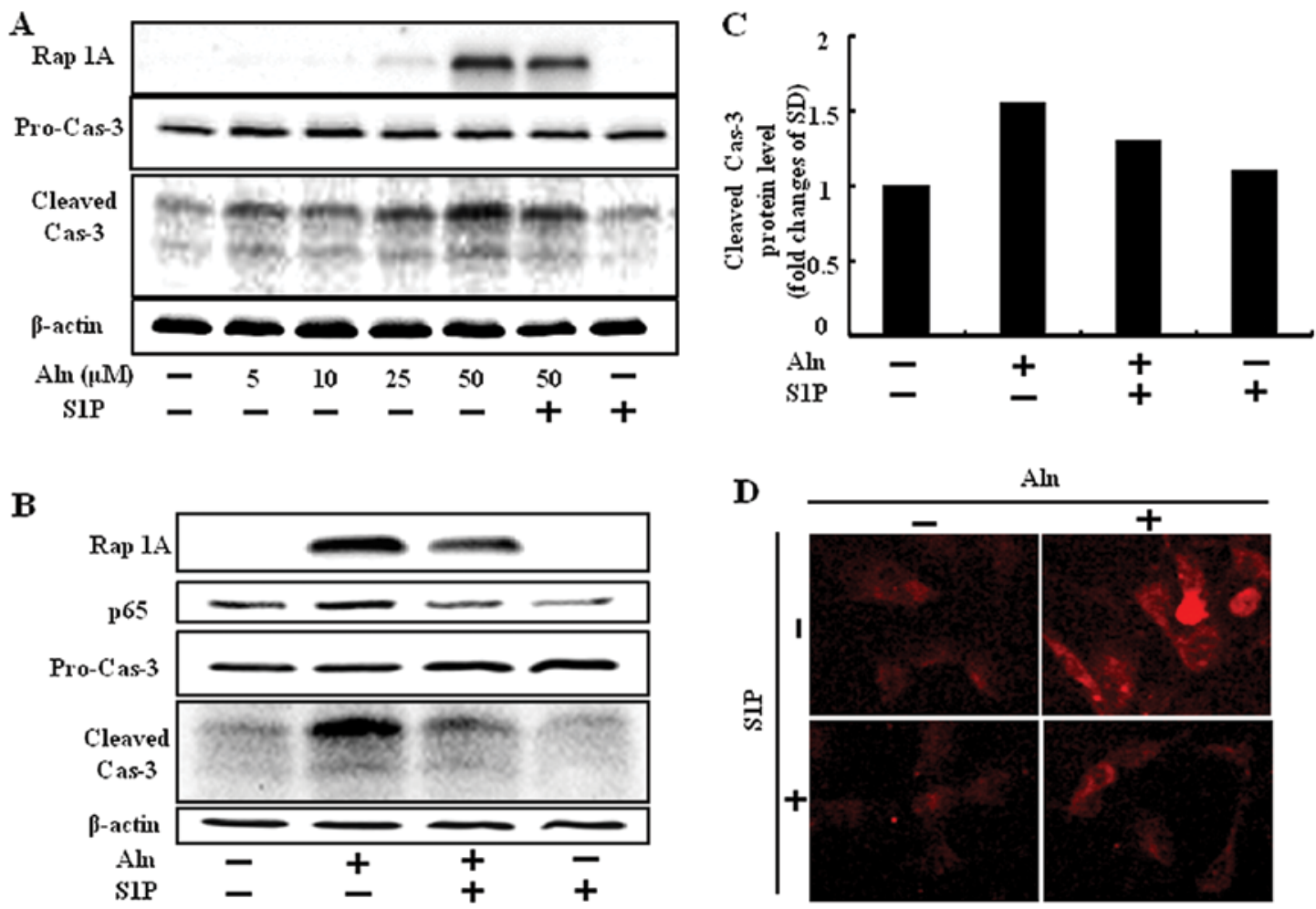

Figure 3. Effects of S1P treatment on BP-induced cleaved caspase-3 and NF-kB activation in HUVECs. (A) HUVECs were pretreated with S1P (1 h) and then exposed to Aln for $12 \mathrm{~h}$ in a dose-dependent manner. The treated cells were assessed for Rap-1A and caspase-3 cleavage by western blot analysis. Results were normalized with $\beta$-actin. (B) The experiments were repeatedly performed to confirm the results. Cells were pretreated with S1P $(1 \mathrm{~h})$ and then exposed to Aln for $12 \mathrm{~h}$. The treated cells were assessed for p65, Rap-1A and caspase-3 cleavage by western blot analysis. Levels of cleaved caspase-3 were normalized to $\beta$-actin (C) The cleaved caspase-3 protein levels in HUVECs treated as described in (A). Expression levels were determined by western blot analysis and densitometric values are shown below the western blot analysis. (D) The treated cells were immunostained with cleaved caspase-3 antibody (red). BP, bisphosphonate; S1P, sphingosine-1-phosphate; Aln, alendronate.

Aln-induced caspase-3 activity with western blotting and immunofluorescence analysis. HUVECs activated caspase-3 cleavage in the treatment of Aln. By contrast, cleaved caspase-3 decreased Aln-induced protein level in the presence of S1P (Fig. 3).

Aln inhibits the cell survival pathway stimulated by the $\mathrm{PI} 3 \mathrm{~K} / \mathrm{Akt} / \mathrm{NF}-\kappa \mathrm{B}$ pathway, thus causing apoptosis of osteosarcoma cells (6), and zoledronic acid induces the activation of NF- $\kappa \mathrm{B}$ in dendritic cells (14). We previously demonstrated that BPs induce cell death in normal endothelial cells and that APC could inhibit BP-mediated endothelial cell damage via inhibition of $N F-\kappa B(13)$. We studied the expression of $\mathrm{NF}-\kappa \mathrm{B}$ in response to $\mathrm{S} 1 \mathrm{P}$, and found that $\mathrm{NF}-\kappa \mathrm{B}$ was markedly increased by Aln in HUVECs and they were blocked in response to S1P treatment (Fig. 3B).

These results confirmed S1P has inhibitory effects on $\mathrm{BP}-$ induced caspase- 3 and $\mathrm{NF}-\kappa \mathrm{B}$ activation.

$S 1 P$ protects $B P$-induced endothelial cell death via inhibition of JNK. JNK belongs to the mitogen-activated protein kinase family and also plays a role in the cellular apoptosis pathway. HUVECs were treated with Aln and the role of p-JNK activity on BP-induced endothelial cell death in the presence or absence of S1P was evaluated by western blotting and immunofluorescence analysis. A significant increase in p-JNK levels was observed in HUVECs treated with Aln. On the contrary, the presence of S1P significantly reduced the level of p-JNK (Fig. 4A-C). The result showed that the level of p-JNK was upregulated by Aln, and S1P inhibited Aln-induced phosphorylation of JNK.

In order to confirm this result, we tested whether SP600125, a JNK inhibitor, was able to inhibit BP-induced endothelial cell death. The inhibitor was added to HUVECs in the presence of $0.5 \mu \mathrm{M}$ S1P for $12 \mathrm{~h}$ at concentrations of $1 \mu \mathrm{M}$. SP600125 attenuated reduced p-JNK activation that was induced by Aln (Fig. 4D and E). Furthermore, SP600125 decreased cleaved caspase-3 (Fig. 4D) and increased the cell viability compared with Aln treatment only (Fig. 4F and G). Therefore, we suggest that S1P protects against BP-induced endothelial cell death via inhibition of JNK.

\section{Discussion}

Bisphosphonates (BPs), stable analogs of pyrophosphate, strongly inhibit bone resorption and have been used to treat various diseases caused by increased bone resorption, such as postmenopausal osteoporosis, and tumor bone metastases (15). They are divided into two groups, according to the structure of the side chains; a nitrogen-containing type and a non-nitrogencontaining type. Non-nitrogen-containing BPs are reported to act through the intracellular accumulation of nonhydrolyzable ATP analogs that exert cytotoxic effects on osteoclasts (16), while nitrogen-containing BPs are known to inhibit the mevalonate pathway and reduce the prenylation of small GTP-binding proteins, such as Ras, Rho, Rac and Cdc42 (17). 
A

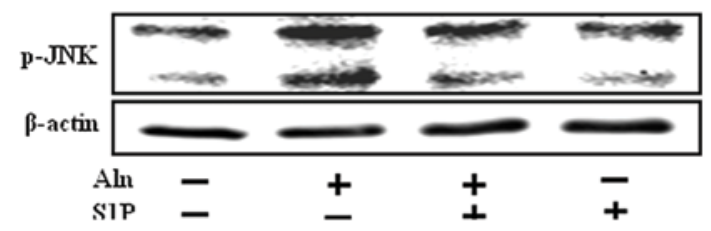

B

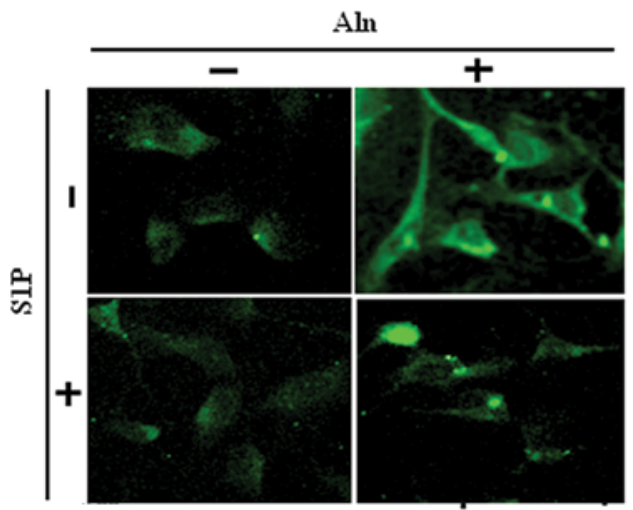

C

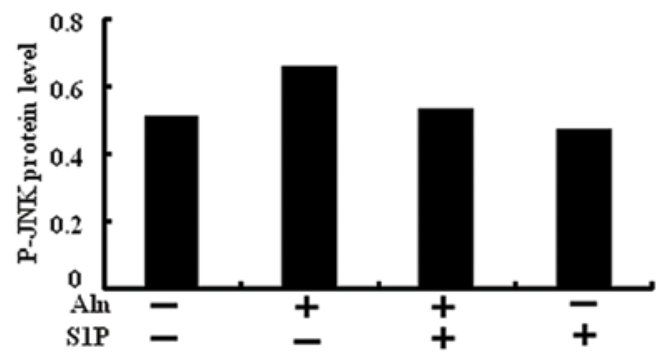

D

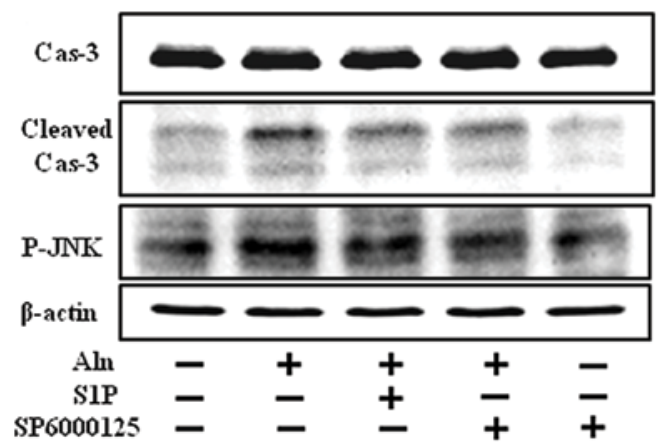

E

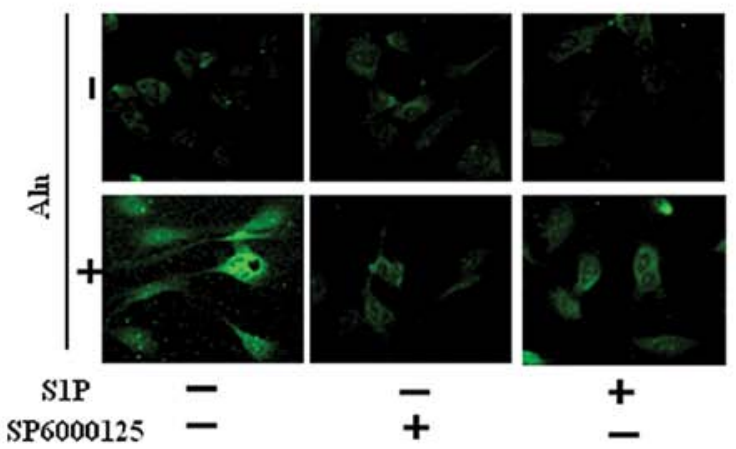

F
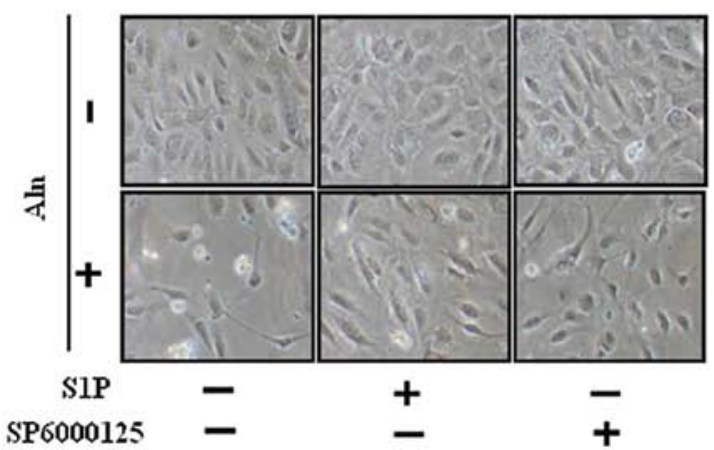

G

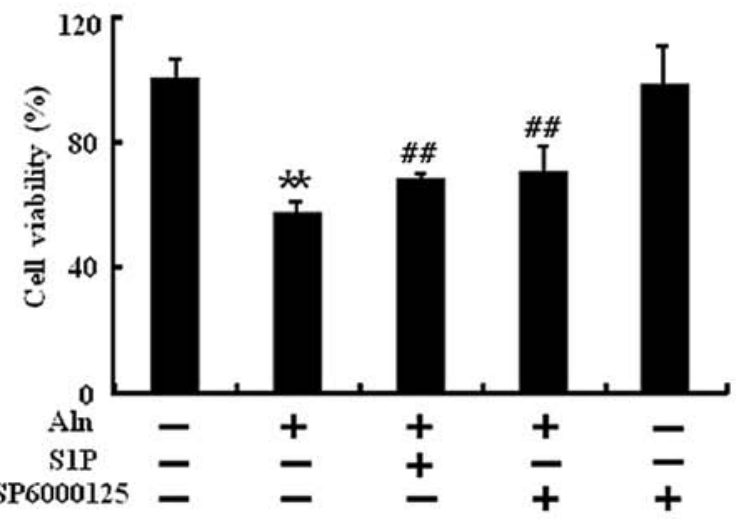

Figure 4. S1P prevents BP-induced HUVEC cell death via regulation of the JNK pathway. (A) HUVECs were pretreated with S1P (1 h) and then exposed to Aln for $12 \mathrm{~h}$. The treated cells were assessed for p-JNK by western blot analysis. (B) The treated cells were immunostained with p-JNK antibody (green). (C) The p-JNK protein levels in HUVECs treated as described in (A). Expression levels were determined by western blot analysis and densitometric values are shown below the western blot analysis. (D) HUVECs were pretreated with $0.5 \mu \mathrm{M} \mathrm{S} 1 \mathrm{P}(1 \mathrm{~h})$ or $1 \mu \mathrm{M} \mathrm{SP} 6000125$ and then exposed to Aln for $12 \mathrm{~h}$. The treated cells were assessed for caspase-3 cleavage and p-JNK by western blot analysis. Results were normalized with $\beta$-actin. (E) The treated cells were immunostained with p-JNK antibody (green). (F) The cell morphology was photographed with a light microscope (x200). (G) Cell viability was measured after Annexin $\mathrm{V}$ assay by flow cytometry. ${ }^{* *} \mathrm{P}<0.01$, significant differences between control and each treatment group. ${ }^{\# \#} \mathrm{P}<0.005$, significant differences vs. BP. S1P, sphingosine-1-phosphate; BP, bisphosphonate; Aln, alendronate.

N-BPs may cause apoptosis by inhibiting the mevalonate pathway and, hence, by preventing protein prenylation $(17,18)$; it is possible that the delay before the appearance of apoptotic cells is dependent on the rate of loss of prenylated proteins that may promote cell survival or maintain normal cell function, such as Ras or nuclear lamins (19). BP has been reported to exert antiangiogenic effects in vivo and in vitro (4). Zoledronate inhibits HUVEC adhesion, survival, migration and it affects ERK1/2, JNK, Rock, FAK and PKB as kinases in a prenylation-depen- dent manner (7). In this study, we confirmed that alendronate induced endothelial cell death through activation of JNK as well as on the caspase- 3 and NF- $\mathrm{KB}$ signaling pathway known to regulate apoptotic cell death in a prenylation-dependent manner (Figs. 3A and B and 4A). However, this mechanism does not fully determine the effects of unprenylated proteins on cell death, and it remains elusive in nitrogen-containing BP-induced endothelial cell apoptosis in connection with unprenylation of protein. 
Sphingolipids are ubiquitous components of cell membranes and their metabolites ceramide (Cer), sphingosine (Sph), and Sphingosine-1-phosphate (S1P). S1P is the terminal product of sphingolipid metabolism, a process that occurs in all mammalian cells. It is formed by the phosphorylation of sphingosine, by two kinases, sphingosine kinase 1 and 2 (SphK1 and SphK2) and its levels are tightly controlled both by the SphKs and the enzymes which include S1P lyase (SPL) (9). S1P in serum is mainly released from stores in activated platelets (20), and, when released, it can interact with endothelial cells thus playing a role in endothelial cell migration, proliferation and angiogenesis. Serum starvation-induced HUVEC cell death is enhanced by inhibiting the intracellular pathway through overexpression of SphK1 (21).

JNK can activate apoptotic signaling either through nuclear or mitochondrial signaling pathways. Activated JNK translocates to the nucleus where it induces transcription of pro-apoptotic genes such as Bax and Bak, and decreases the expression of pro-survival genes including c-Jun (22). The sphingolipid degradation product trans-2-hexadecenal induces cytoskeletal reorganization and apoptosis in a JNK-dependent manner (22). S1P is known for relevant bioactive lipid in functions, such as endothelial cell proliferation, adhesion and inhibition of permeability.

In this study, we found that $\mathrm{S} 1 \mathrm{P}$ induces a decrease in the expression of cleaved caspase- 3 and NF- $\kappa \mathrm{B}$ in HUVECs. Caspase -3 cleavage and $N F-\kappa B$ are members of the activator of apoptosis protein family that is expressed in BP-induced endothelial cell death (13). BPs also cause apoptosis and activate JNK signaling pathways in osteoclasts and J774 macrophages in vitro (23). Collectively, we suggested that BP-induced apoptosis is accompanied by activation of JNK in endothelial cells and S1P, the terminal product of sphingolipid metabolism, prevents JNK activation. Furthermore, S1P attenuated the BP-induced apoptosis of HUVECs by inhibition of JNK (Fig. 4) and we suggest that S1P has the potential to be a therapeutic drug in various vascular diseases induced by endothelial cell damage. Further studies are required to improve the cotreatment with S1P and BP conferring endothelial protection with few side-effects in various therapies.

\section{Acknowledgements}

This study was supported by the National Research Foundation of the Korea Grant funded by the Korean Government (2010-E00019, 2010-21492).

\section{References}

1. Roelofs AJ, Thompson K, Gordon S and Rogers MJ: Molecular mechanisms of action of bisphosphonates: current status. Clin Cancer Res 12: 6222s-6230s, 2006.

2. Allegra A, Alonci A, Penna G, et al: Bisphosphonates induce apoptosis of circulating endothelial cells in multiple myeloma patients and in subjects with bisphosphonate-induced osteonecrosis of the jaws. Acta Haematol 124: 79-85, 2010.

3. Tassone P, Tagliaferri P, Viscomi C, et al: Zoledronic acid induces antiproliferative and apoptotic effects in human pancreatic cancer cells in vitro. Br J Cancer 88: 1971-1978, 2003.

4. Hashimoto K, Morishige K, Sawada K, et al: Alendronate suppresses tumor angiogenesis by inhibiting Rho activation of endothelial cells. Biochem Biophys Res Commun 354: 478-484, 2007.
5. Fu L, Tang T, Miao Y, Zhang S, Qu Z and Dai K: Stimulation of osteogenic differentiation and inhibition of adipogenic differentiation in bone marrow stromal cells by alendronate via ERK and JNK activation. Bone 43: 40-47, 2008.

6. Inoue R, Matsuki NA, Jing G, Kanematsu T, Abe K and Hirata M: The inhibitory effect of alendronate, a nitrogen-containing bisphosphonate on the PI3K-Akt-NFkappaB pathway in osteosarcoma cells. Br J Pharmacol 146: 633-641, 2005.

7. Hasmim M, Bieler G and Ruegg C: Zoledronate inhibits endothelial cell adhesion, migration and survival through the suppression of multiple, prenylation-dependent signaling pathways. J Thromb Haemost 5: 166-173, 2007.

8. Tsai SH, Huang PH, Chang WC, et al: Zoledronate inhibits ischemia-induced neovascularization by impairing the mobilization and function of endothelial progenitor cells. PLoS One 7: e41065, 2012.

9. Maceyka M,Harikumar KB, Milstien S and Spiegel S: Sphingosine1-phosphate signaling and its role in disease. Trends Cell Biol 22: 50-60, 2012.

10. Morales-Ruiz M,Lee MJ,Zollner S, et al: Sphingosine 1-phosphate activates Akt, nitric oxide production, and chemotaxis through a Gi protein/phosphoinositide 3-kinase pathway in endothelial cells. J Biol Chem 276: 19672-19677, 2001.

11. Ishii $M$ and Kikuta J: Sphingosine-1-phosphate signaling controlling osteoclasts and bone homeostasis. Biochim Biophys Acta 1831: 223-227, 2013.

12. Kimura T, Sato K, Kuwabara A, et al: Sphingosine 1-phosphate may be a major component of plasma lipoproteins responsible for the cytoprotective actions in human umbilical vein endothelial cells. J Biol Chem 276: 31780-31785, 2001.

13. Seol JW, Lee YJ, Jackson CJ, Sambrook PN and Park SY: Activated protein $\mathrm{C}$ inhibits bisphosphonate-induced endothelial cell death via the endothelial protein $\mathrm{C}$ receptor and nuclear factor- $\kappa$ B pathways. Int J Mol Med 27: 835-840, 2011.

14. Wolf AM, Rumpold H, Tilg H, Gastl G, Gunsilius E and Wolf D: The effect of zoledronic acid on the function and differentiation of myeloid cells. Haematologica 91: 1165-1171, 2006.

15. Russell RG, Watts NB, Ebetino FH and Rogers MJ: Mechanisms of action of bisphosphonates: similarities and differences and their potential influence on clinical efficacy. Osteoporos Int 19: 733-759, 2008.

16. Rogers MJ, Russell RG, Blackburn GM, Williamson MP and Watts DJ: Metabolism of halogenated bisphosphonates by the cellular slime mould Dictyostelium discoideum. Biochem Biophys Res Commun 189: 414-423, 1992.

17. Luckman SP, Hughes DE, Coxon FP, Graham R, Russell G and Rogers MJ: Nitrogen-containing bisphosphonates inhibit the mevalonate pathway and prevent post-translational prenylation of GTP-binding proteins, including Ras. J Bone Miner Res 13: 581-589, 1998.

18. Luckman SP, Coxon FP, Ebetino FH, Russell RG and Rogers MJ: Heterocycle-containing bisphosphonates cause apoptosis and inhibit bone resorption by preventing protein prenylation: evidence from structure-activity relationships in J774 macrophages. J Bone Miner Res 13: 1668-1678, 1998.

19. Perez-Sala D and Mollinedo F: Inhibition of isoprenoid biosynthesis induces apoptosis in human promyelocytic HL-60 cells. Biochem Biophys Res Commun 199: 1209-1215, 1994.

20. Yatomi Y, Igarashi Y, Yang L, et al: Sphingosine 1-phosphate, a bioactive sphingolipid abundantly stored in platelets, is a normal constituent of human plasma and serum. J Biochem 121: 969-973, 1997.

21. Limaye V, Li X, Hahn C, et al: Sphingosine kinase-1 enhances endothelial cell survival through a PECAM-1-dependent activation of PI-3K/Akt and regulation of Bcl-2 family members. Blood 105: 3169-3177, 2005.

22. Kumar A, Byun HS, Bittman R and Saba JD: The sphingolipid degradation product trans-2-hexadecenal induces cytoskeletal reorganization and apoptosis in a JNK-dependent manner. Cell Signal 23: 1144-1152, 2011.

23. Dunford JE, Rogers MJ, Ebetino FH, Phipps RJ and Coxon FP: Inhibition of protein prenylation by bisphosphonates causes sustained activation of Rac, Cdc42, and Rho GTPases. J Bone Miner Res 21: 684-694, 2006. 